\title{
Role of Dyestuff in Improving Dye-Sensitized Solar Cell Performance
}

\author{
Yehia Selim ${ }^{1}$, Ahmed A. Mohamed ${ }^{2}$ \\ ${ }^{1}$ Faculty of Engineering, Pharos University, Alexandria, Egypt \\ ${ }^{2}$ Faculty of Engineering, Menoufia University, Shebin El-Kom, Egypt \\ yehia.selim@pua.edu.eg, Ameny_49@yahoo.com
}

\begin{abstract}
Dye-sensitized solar cells DSSCs have attracted great attention for their simple fabrication process, low production costs, relatively high conversion efficiency, and being environmental friendly.
\end{abstract}

DSSC are a combination of materials, consisting of a transparent electrode coated with a dye-sensitized mesoporous film of nanocrystalline particles of $\mathrm{TiO} 2$, an electrolyte containing a suitable redox-couple and a electrode.

DSSCs use organic dye assist to produce electricity in a wide range of light conditions, indoors and outdoors. The dye in the solar cell is the key element since it is responsible for light harvesting ability, photoelectron generation (the creation of free charges after injection of electrons into the nanostructured semi-conducting oxide) and electron transfer.

For this reason, this paper gives a background of dyestuff, types and limitations. The motivation of this work is to design a simple, easy and prepare an efficient organic dye sensitizer.

Also, this paper investigates the important criteria which are considered for selecting dye to enhance DSSC efficiency.

Keywords - dye-sensitized solar cells, DSSC performance, dye, poto sensitizers, organic dye sensitizer.

\section{INTRODUCTION}

Solar energy provides a clean, renewable and cheaper energy source for human race, while serving as a primary energy source for another type of energy sources, namely; wind energy, water, bio-energy and fossil fuel. The solar cells used in harvesting the solar power are commonly categorized into different types in respect to the composition of their material e.g. organic dye solar cells, non-crystal, multiple crystal, and single crystal silicon solar cells. A solar cell usually signifies the cell that is made from silicon crystal material. Nevertheless, the production cost of the solar cells based on silicon crystal material compared to the Dye-Sensitized Solar Cells (DSSCs) is high. DSSCs are devices that convert solar to electric energy by light sensitization established on wide energy band semiconductor [1]. DSSC shows a very promising future in the field of photovoltaic cells. DSSC also known as Grätzel cell is a new type of solar cell [2], and have attracted a great interest due to their minimal production cost, and environmental friendliness

DSSC considered as third generation solar cells of high efficiency with low production costs. Although present-day DSSCs provide light-to-electricity conversion of less than $12 \%$, further condensed investigations daily done for improvement this ratio by modifying not only each single component's function of the cell but also optimizing their compilation for easier and efficient communication between them to produce maximum benefits.

In addition, DSSC devices can be manufactured to be semi-transparent to harvest light from any direction in order to be used as photovoltaic windows in buildings or small devices.

The dyestuffs which are the backbone of the DSSC or photo-electro-chemical-voltaic cells are common issue in many research centers interested in developing photovoltaic cells.

This review article presents what we know and what we need to know about role of dye in DSSC in order to formulate basic guidelines and strategies for improving of dye-sensitized solar cells. 
Also, in this perspective article, some important points will be recommended for further increase of DSSC efficiency.

In order to be familiar with the function or dyestuff in the DSSC a concise overview will be summarized about different types of dyestuff, its role in DSSC, relation with colors and light, classification according to application and structure, synthesis and what factors affecting its quality.

\section{DYE SOLAR SENSITIZED CELLS (DSSC) CONSTRUCTION}

DSSC comprises of a nano-crystalline porous semiconductor electrode-absorbed dye as a power electrode and counter electrode, an electrolyte containing iodide and triiodide ions, and shown in Figure (1).

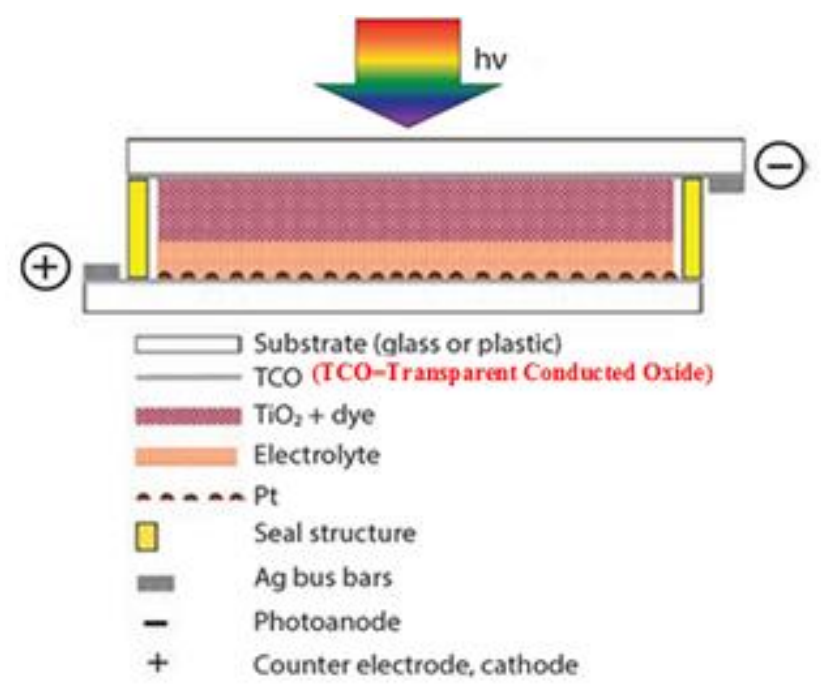

Fig .1. Construction of DSSC

\section{SIMILARITIES OF DSSCS AND PHOTOSYNTHESIS}

Both DSSCs and plants both harvest energy from sunlight. Photovoltaic solar cells collect sunlight and change it to electricity. Plant leaves gather sunlight and convert it into stored chemical energy. Both DSSCs and plants are utilizing dye to convert light energy into another sorts of energy, but they do it in different ways.

Photosynthesis, the effectiveness of the light conversion in plant's leaves in which chlorophyll acts as the dye corresponds to the sensitizer dye in DSSC as shown in Figure (2).

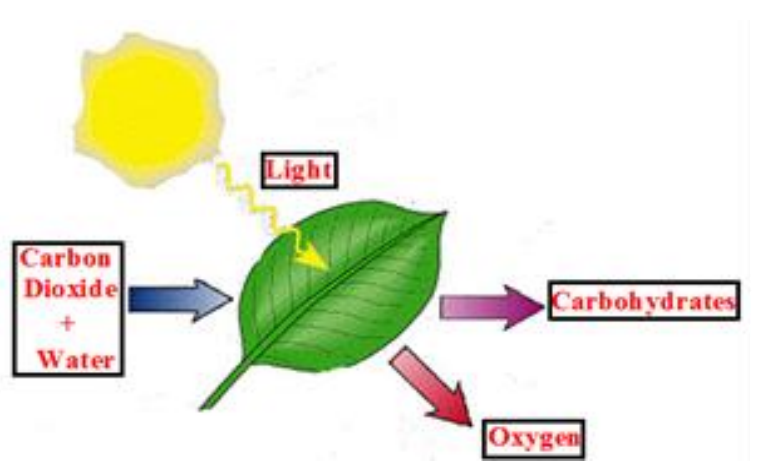

Fig .2.

Light energy transfers (in plant) into chemical reaction forming formal dehyde (from $\mathrm{CO} 2+\mathrm{H} 2 \mathrm{O}+\mathrm{hv}=\mathrm{HCHO}$ $+\mathrm{O} 2)$ which polymerized to glucose $(6 \mathrm{HCHO}=$ $\mathrm{C} 6 \mathrm{H} 12 \mathrm{O} 6$ )then starch ( $66 \mathrm{H} 10 \mathrm{O} 5) \mathrm{n}$ or cellulose polymers, while Light energy converted to electric current in DSSC.

So, the DSSC is designed based on photosynthesis phenomena.

\section{DYE AS PHOTO SENSITIZER}

In spite of several elements combining the DSSC (2 electrodes, dye, electrolyte, sealing),

The dye which acts as sensitizers in DSSCs plays an essential task in absorption and conversion of incident light ray into electricity.

Dye is considered as a promising element for getting more performance to DSSC.

\section{WHAT IS DYE}

By definition Dyes can be said to be colored, ionising and aromatic organic compounds which shows an affinity towards the substrate to which it is being applied. It is generally applied in a solution that is aqueous. Dyes may also require a mordant for better fastness on the material on which it is applied.

Dyes are applied to numerous substrates for example to textiles, leather, plastic, paper etc. in liquid form. One characteristic of dye is that the dyes must get completely or at least partially soluble in which it is being put to. The rule that we apply to other chemicals is similarly applicable to dyes also. Precautions should be taken upon handling of dyes For example certain kind of dyes can be toxic, carcinogenic and can pose as a hazard to health. 


\section{CLASSIFICATION OF DYES}

There are several ways for classification of dyes. It should be noted that each class of dye has a very unique chemical structure and particular way of bonding. While some dyes can react chemically with the substrates forming strong bonds in the process, others can be held by physical forces. One of the prominent ways of several classifications which is more effective to our application is Chemical classification- Based on the nature of chemical structure.

\section{WHY DYES ARE COLORED}

Dyes possess color because they

1. Absorb light in the visible spectrum $(400-700 \mathrm{~nm})$,

2. Have at least one chromophore (color-bearing chemical group),

3. Have a conjugated system, i.e. a structure with alternating double and single bonds, and

4. Exhibit resonance of electrons, which is a stabilizing force in organic compounds.

When any one of these features is lacking from the molecular structure the color is lost.

In addition to chromophores, most dyes also contain groups known as auxochromes (color developers), examples of which are carboxylic acid, sulfonic acid, amino, Nitro, and hydroxyl groups. While these are not responsible for color, their presence can shift the color of a colorant and they are most often used to influence dye solubility.

Table 1 shows the relationships between wavelength of visible (observed) color and absorbed color (Complementary colors).

Table 1. Wavelength of light absorption versus color in organic dyes

\begin{tabular}{|c|c|c|}
\hline $\begin{array}{c}\text { Wavelength } \\
\text { Absorbed }(\mathbf{n m})\end{array}$ & Color Observed & Color Absorbed \\
\hline $400-435$ & Yellow- Green & Violet \\
\hline $435-480$ & Yellow & Blue \\
\hline $480-490$ & Orange & Green-Blue \\
\hline $490-500$ & Red & Blue-Green \\
\hline $500-560$ & Purple & Green \\
\hline $560-580$ & Violet & Yellow- Green \\
\hline $580-595$ & Blue & Yellow \\
\hline $595-605$ & Green-Blue & Orange \\
\hline $605-700$ & Blue-Green & Red \\
\hline
\end{tabular}

\section{THE IMPORTANT FACTORS TO BE CONSIDERED WHEN DESIGNING A DYE.}

When designing a dye for DSSC application, there are many important factors that need to be taken into consideration.

1. The dye should have a broad absorption spectrum, preferably all the way into the near-IR in order to harvest as many incident photons as possible.

When we talk about light and dye we concentrate on $600 \mathrm{~nm}$ out of $1014 \mathrm{~nm}$ wave length or $103 \mathrm{~Hz}$ frequency out of $1014 \mathrm{~Hz}$. As it shown in Figure (3) by part of UV, all visible light and near Infra-red (NIR) range.

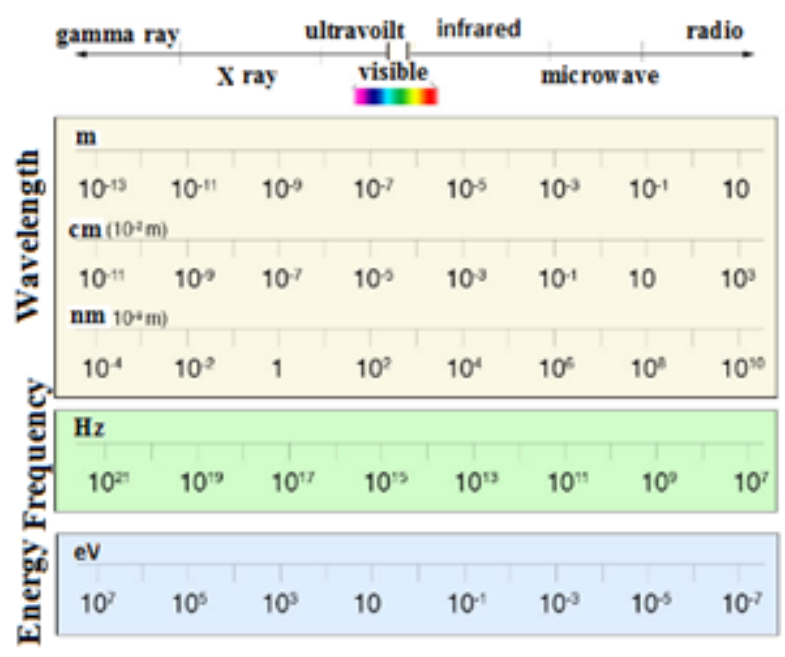

Fig .3. The Electromagnetic Spectrum

2. A high extinction coefficient will enable the use of thinner semiconductor films and still keep a high degree of absorbed photons as shown in Figure(4).

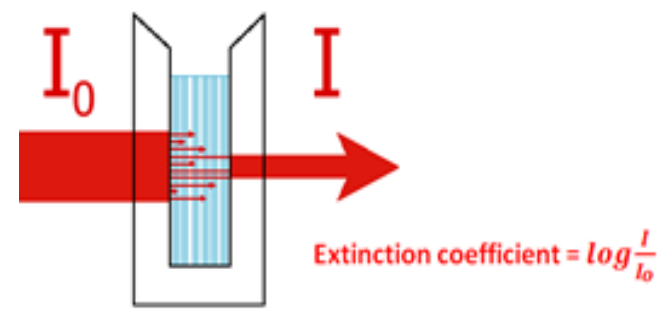

Fig .4.

3. It must bind strongly to the semiconductor surface for long term stability.

4. The energy levels should match the conduction band of the semiconductor and the redox potential of the hole-conductor, as shown in Figure (5). 
5. Easy and straightforward synthesis for future large scale production.

6. Low toxicity and possibility to recycle.

7. High photo-stability to sustain at least 20 years of use.

8. Achieve a long lifetime of the injected electrons by blocking the recombination pathways.

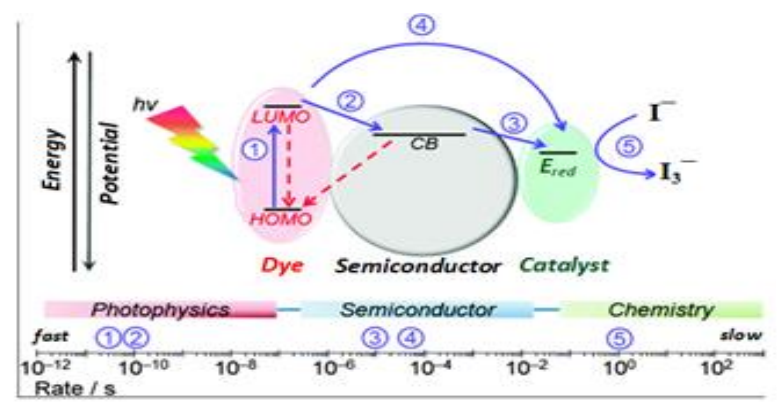

Fig .5.

The properties of the organic sensitizers can be modified by incorporating different groups into the molecule.

By choosing the right design, the sensitizer can be tuned in order to increase the long wavelength absorption, achieve a high extinction coefficient, and shift the energy levels to improve the performance of the solar cell.

The positions of the energy levels in the sensitizer are of great importance.

A small gap between the levels gives the possibility to harvest more low energy photons.

If the energy exceeds the band gap of the semiconductor, the photon can be absorbed and an electron will be excited from the valence band to the conduction band. Electrons in the conduction band can move, which allows for electric current, as shown in Figure (6). The difference between the photon energy and the band gap will end up as heat.

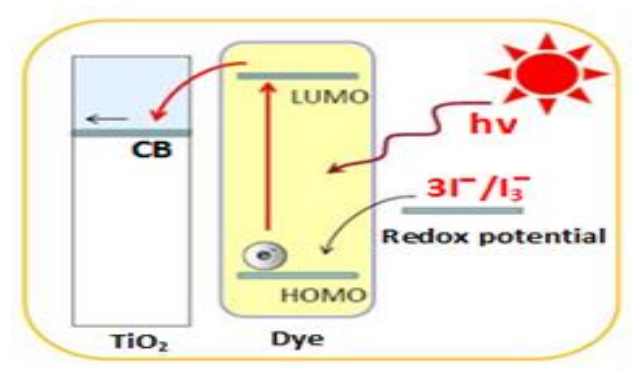

Fig .6.
A common way of designing organic dyes is the D-mA strategy [4], where the molecule is built up of an electron donor, a conjugated linker, and an electron acceptor, as illustrated in Figure (7). This structure will yield an intramolecular charge separation upon excitation which is desired for DSSCs.

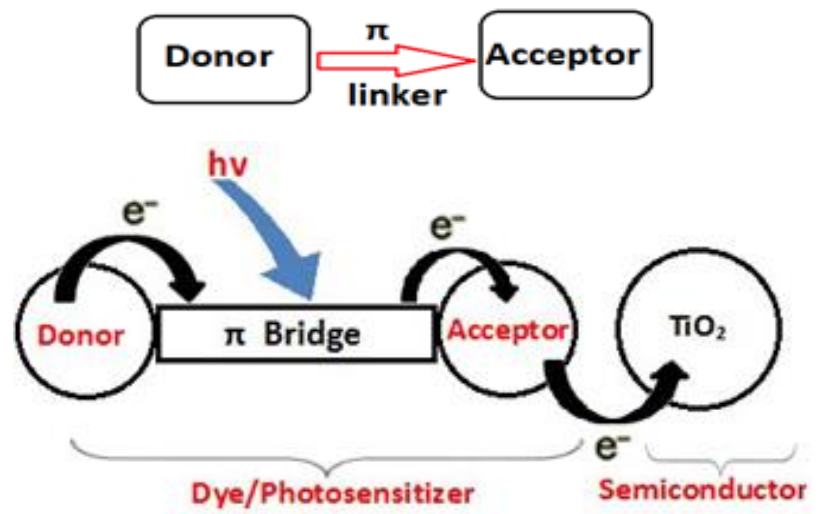

Fig .7.

\section{CONCLUSION}

This review article presents what we know and what we need to know about role of dye in DSSC in order to formulate basic guidelines and strategies for improving of dye-sensitized solar cells.

Also, this paper investigates the important criteria which are considered for selecting dye to enhance DSSC efficiency, and it has been shown that, there are a great number of factors that need to be kept in mind.

\section{REFERENCES}

[1] Oluwaseun Adedokun et al., Review on Natural Dye-Sensitized Solar Cells, International Journal of Engineering Technology Vol. 2, No.2, 2016.

[2] B. O'Regan, M. Gratzel, (1991) A Low-cost high efficiency solar cell based on dye-sensitized colloidal TiO2 films, Nature, 353: 737-740.

[3] http://www.globalspec.com/reference/41766/2032 79/classification-of-dyes.

[4] Enediyne as $p$ linker in D-p-A dyes for dyesensitized solar cells $†$ Youfu Wang,a Luhua Dong,a Zhiwei Zheng,b Xing Li,b Rulin Xiong,a Jianli Hua*b and Aiguo Hu*a, RSC Advances Issue 15, 2016 (An international journal to further the chemical sciences). 